\title{
A IMPORTÂNCIA DA AVALIAÇÃO DA SATISFAÇÃO DOS USUÁRIOS DO SERVIÇO DE FISIOTERAPIA: REVISÃO NARRATIVA
}

Samuel de Sousa Ribeiro, Júlio Ribeiro Bravo Gonçalves Junior. A importância da avaliação da satisfação dos usuários do serviço de fisioterapia: revisão narrativa. Revista Saúde Dinâmica, vol. 2, núm.3, 2020. Faculdade Dinâmica do Vale do Piranga. 


\title{
A importância da avaliação da satisfação dos usuários do serviço de fisioterapia: revisão narrativa
}

\section{The importance of evaluating the satisfaction os users os the physiotherapy servisse: narrative review}

Samuel de Sousa Ribeiro1, Júlio Ribeiro Bravo Gonçalves Junior2

${ }^{I}$ Discente do Curso de Fisioterapia, Faculdade Dinâmica do Vale do Piranga, ORCID: 0000-0002-2570-5774

${ }^{2}$ Docente no Curso de Fisioterapia, Faculdade Dinâmica do Vale do Piranga, ORCID: 0000-0002-6989-7835

Autor correspondente: samuelsousaribeiro@hotmail.com

\section{Resumo}

Um dos aspectos fundamentais da qualidade de um serviço relaciona-se à satisfação de seu usuário. A preocupação com a qualidade nos serviços de fisioterapia é uma crescente, tendo como base o aumento do nível de exigência de parte da sociedade. O objetivo dessa revisão foi identificar, reunir e analisar os artigos científicos que dissertam sobre a satisfação dos usuários no serviço de fisioterapia. Foi realizado uma seleção de artigos por meio eletrônico em abril de 2020 com estudos publicados nos últimos 12 anos (2008-2020) nas bases de dados PubMed, SciELO e PEDro, no idioma inglês. Foram selecionados 5 estudos para a revisão narrativa. De modo geral todos os artigos corroboram a ideia de que avaliação da satisfação dos usuários do serviço de fisioterapia é importante para a determinação e qualidade do serviço. Através desse conhecimento dos fatores relacionados a satisfação e percepção dos usuários do serviço de fisioterapia, os gestores, administradores e profissionais podem buscar novos modelos organizacionais com o foco na melhora da qualidade do serviço.

Palavras-chave: Avaliação, Usuário de fisioterapia e Satisfação.

\begin{abstract}
One of the fundamental aspects of the quality of a service is related to the satisfaction of its user. The concern with the quality of physiotherapy services is growing, based on the increase in the level of demand from part of society. The objective of this review was to identify, collect and analyze scientific articles that discuss user satisfaction in the physiotherapy service. A selection of articles was carried out electronically in April 2020 with studies published in the last 12 years (2008-2020) in the PubMed, SciELO and PEDro databases, in English. Five studies were selected for the narrative review. In general, all articles corroborate the idea that assessing the satisfaction of users of the physiotherapy service is important for the determination and quality of the service. Through this knowledge of factors related to the satisfaction and perception of users of the physical therapy service, managers, administrators and professionals can seek new organizational models with a focus on improving the quality of the service.
\end{abstract}

Key words: Evaluation, Physical Therapy, User e Satisfaction. 


\section{INTRODUÇÃO}

Um dos aspectos fundamentais da qualidade de um serviço relaciona-se à satisfação de seu usuário. Esse cuidado nos serviços de saúde é uma crescente, tendo como base o aumento do nível de exigência de parte da sociedade. Serviços eficazes, seguros e que geram resultados satisfatórios são itens que estão diretamente relacionados a percepção de qualidade (MAIA, et al., 2011).

A Fisioterapia é uma ciência que estuda, previne e trata distúrbios cinéticos funcionais e apenas recentemente tem desenvolvido ferramentas que avaliam como sua pratica é percebida pelos pacientes (BRITO, JESUS e FERNANDES, 2012). A avaliação da percepção dos serviços de saúde passou a ser discutida, com maior ênfase, a partir da segunda metade do século 20, quando começaram os estudos sobre a avaliação da assistência em saúde, com foco na qualidade do atendimento (MACHADO e NOGUEIRA, 2008).

$\mathrm{Na}$ área da saúde, esta avaliação torna-se mais difícil pelo fato de poucas pessoas obterem conhecimento técnico do funcionamento da saúde pública (BATESON e HOFFMAN, 2003). Se tratando na área da Fisioterapia a dificuldade aumenta, pois um atendimento fisioterapêutico comumente demanda muito mais tempo que uma visita clínica rotineira, envolvendo contato físico, e muitas das vezes a terapia requer participação ativa do paciente (MENDONÇA e GUERRA, 2007). A fisioterapia sendo habitualmente uma profissão em que se exige bastante contato com o paciente e diversas vezes participação ativa do mesmo salienta características que podem influenciar a sua satisfação (RODRIGUES, RAIMUNDO e SILVA, 2010).

O consumo de um serviço de saúde está associado às exigências e expectativas vinculadas a um problema de saúde do usuário, assim, após o uso desses serviços, o paciente poderá declarar se suas necessidades e expectativas foram alcançadas e resolvidas. O nível de percepção interfere na qualidade nos diversos momentos, desde a entrada do paciente, durante o atendimento, até a saída do serviço de saúde (SANTIAGO et al., 2013; MENDES et al., 2009).

Quando há determinação de avaliar a qualidade de um serviço na área da saúde, o primeiro passo é seletar um índice para servir de parâmetro, podendo ser o próprio serviço de saúde, o usuário ou o profissional (DONABEDIAN, 1988). Portanto uma ideia lógica é que a 
avaliação da qualidade deve basear-se na percepção dos usuários porquê ele é um importante indicador de qualidade do serviço prestado e permite avaliar a relação profissional-paciente (ASSUNÇÃO, SANTOS e GIGANTE, 2001).

Porém a satisfação do usuário é multidimensional, podendo ele estar satisfeito em vários aspectos da assistência e insatisfeito simultaneamente com outros (BEATTIE, 2002). Pois com o paciente visto como um cliente dos serviços de saúde, ele pode se colocar como usuário, avaliando os serviços do ponto de vista do consumidor (aspectos individuais), e como cidadão, levando em conta a sociedade como um todo. Como cliente, pode querer um tempo de espera menor para o atendimento mais rápido e eficaz, já como cidadão, pode querer que todas as pessoas sejam atendidas com cautela, implicando um tempo de espera maior (VAITSMAN, 2005).

Dessa forma, ao avaliar a qualidade, os pacientes fazem comparações entre a atuação do serviço sobre suas expectativas, os resultados são agradáveis quando a qualidade percebida é alcançada. Essa qualidade pode ser notada através de fatores palpáveis (aquilo que é de caráter pessoal) e impalpável (ligados à relação externas). De forma global, observa-se que a percepção dos usuários é respaldada mais nos aspectos individuais do que técnicos (FADEL e REGIS FILHO, 2009; GURGEL JUNIOR e VIEIRA, 2002).

Como a satisfação do paciente pode ou não interferir na criação de vínculos, no processo do cuidado, na percepção da qualidade e no seu desfecho final é necessário o aumento de pesquisas que visam à avaliação dos serviços prestados.

O objetivo dessa revisão foi identificar, reunir e analisar os artigos científicos que dissertam sobre a importância da satisfação dos usuários no serviço de fisioterapia.

\section{MATERIAIS E MÉTODOS}

Trata-se de uma revisão narrativa. Para direcionar o presente estudo buscou-se responder a seguinte questão norteadora: "como se apresenta na literatura científica a importância da avaliação da satisfação dos usuários do serviço de fisioterapia”. Os dados foram coletados em abril de 2020 por meio eletrônico nas bases de dados PubMed, SciELO e PEDro, no idioma inglês. Para pesquisa utilizaram-se as seguintes palavras-chave: evaluation, physical 
therapy, user e satisfaction. Os critérios de inclusão dos artigos buscaram selecionar estudos descritivos que dissertavam sobre a importância da avaliação da satisfação dos usuários do serviço de fisioterapia e publicados nos últimos 12 anos (2008-2020) no idioma português ou inglês. A escolha foi feita através do título e resumo dos artigos quando necessário leitura completa. Excluíram-se artigos duplicados, revisões de literatura e estudos que não se relacionavam com o tema desse trabalho. Foram encontrados um total de 123 artigos. Foram excluídos um total de 118 artigos que não tinham relação com o tema ou não se encaixavam nos critérios exigidos. Sendo assim foram selecionados 5 estudos para a revisão sistemática conforme descrito na Figura 1.

Figura 1- Fluxograma descrevendo como foi realizada a seleção dos artigos para revisão narrativa.

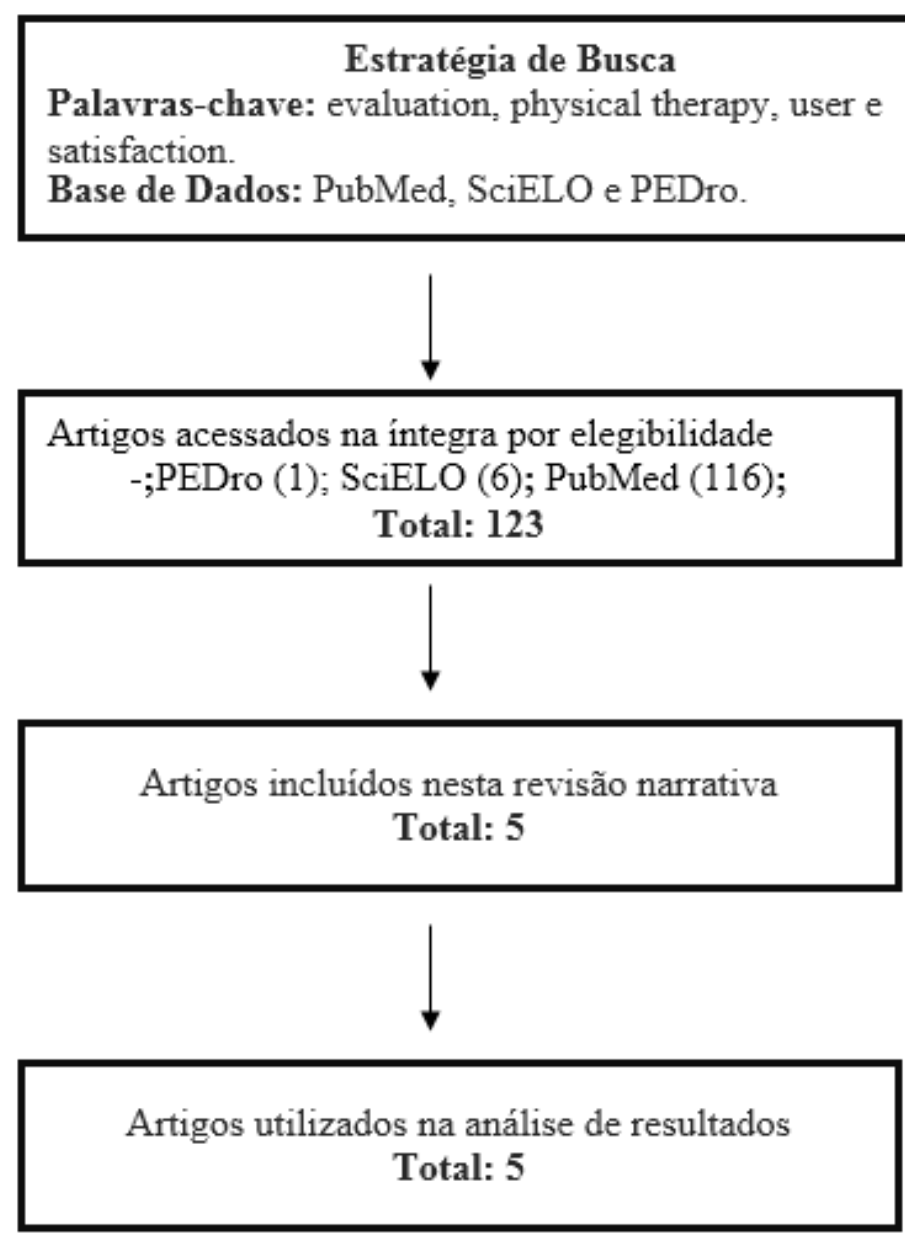

Artigos excluídos após leitura do título e resumo (Falta de relação com o tema da revisão/ artigos repetidos)

Total: 118 


\section{RESULTADOS E DISCUSSÃO}

Nesta revisão narrativa foram selecionados 5 artigos que abordavam sobre o tema proposto. Esses artigos foram apresentados no Quadro 1 e agrupados de acordo com a metodologia e os resultados obtidos, conforme demonstrado no Quadro 2.

Quadro 1- Publicações selecionadas para análise do conteúdo

\begin{tabular}{|l|l|}
\hline AUTOR/ANO & TÍTULO \\
\hline $\begin{array}{l}\text { Machado et al. } \\
(2008)\end{array}$ & $\begin{array}{l}\text { Avaliação da satisfação dos usuários de serviços de } \\
\text { Fisioterapia }\end{array}$ \\
\hline $\begin{array}{l}\text { Frez et al. } \\
(2011)\end{array}$ & $\begin{array}{l}\text { Satisfação dos usuários dos serviços ambulatoriais de } \\
\text { fisioterapia da rede pública }\end{array}$ \\
\hline $\begin{array}{l}\text { Almeida et al. } \\
(2013)\end{array}$ & $\begin{array}{l}\text { Análise do nível de satisfação do usuário em um serviço } \\
\text { público de fisioterapia. }\end{array}$ \\
\hline $\begin{array}{l}\text { Silva et al. } \\
(2014)\end{array}$ & $\begin{array}{l}\text { Fisioterapia ambulatorial na rede pública de saúde de Campo } \\
\text { Grande (MS, Brasil) na percepção dos usuários: resolutividade } \\
\text { e barreiras }\end{array}$ \\
\hline $\begin{array}{l}\text { Cornec et al. } \\
(2019)\end{array}$ & $\begin{array}{l}\text { Determinantes da satisfação com a reabilitação motora em } \\
\text { pessoas com paralisia cerebral: uma pesquisa nacional na } \\
\text { França (ESPaCe). }\end{array}$ \\
\hline
\end{tabular}

Quadro 2- Caracterização dos estudos quanto ao objetivo, tipo de estudo e as contribuições (resultados/conclusão) traduzidos para o idioma português.

\begin{tabular}{|c|c|c|c|}
\hline $\begin{array}{l}\text { AUTOR } \\
(\text { ANO) }\end{array}$ & OBJETIVO & $\begin{array}{l}\text { TIPO } \\
\text { ESTUDO }\end{array}$ & CLUSÃO \\
\hline $\begin{array}{l}\text { Machado } \\
\text { et al. } \\
(2008)\end{array}$ & $\begin{array}{l}\text { O objetivo foi avaliar a } \\
\text { satisfação dos usuários } \\
\text { dos serviços de } \\
\text { Fisioterapia em } \\
\text { Teresina e caracterizar } \\
\text { o perfil } \\
\text { sociodemográfico } \\
\text { desses indivíduos. }\end{array}$ & $\begin{array}{l}\text { Trata-se de uma } \\
\text { pesquisa } \\
\text { descritiva, } \\
\text { transversal. } \\
\begin{array}{l}\text { População } \\
\text { avaliada: } \\
\text { amostra }\end{array} \text { A } \\
\text { probabilística, }\end{array}$ & $\begin{array}{l}\text { Os usuários eram maioria do } \\
\text { gênero feminino com média de } \\
\text { idade de } 49 \text { anos, casados, } \\
\text { analfabetos, com renda familiar } \\
\text { entre um e três salários mínimos e } \\
\text { SUS-dependentes. O acesso foi } \\
\text { considerado fácil, o meio de } \\
\text { locomoção mais utilizado foi o }\end{array}$ \\
\hline
\end{tabular}




\begin{tabular}{|c|c|c|c|}
\hline & & $\begin{array}{l}\text { estratificada } \\
\text { proporcional, } \\
\text { constituída por } 376 \\
\text { usuários com idade } \\
\text { superior a } 18 \text { anos. }\end{array}$ & $\begin{array}{l}\text { coletivo sendo a média para } \\
\text { chegar no local do atendimento de } \\
28 \text { minutos. Sobre o atendimento } \\
\text { foi relatado que a marcação de } \\
\text { consulta e o tempo de espera são } \\
\text { os problemas encarados nos } \\
\text { serviços das rede municipal e } \\
\text { estadual. A indicação médica } \\
\text { precede a escolha do serviço, os } \\
\text { usuários confiavam no } \\
\text { atendimento recebido e os } \\
\text { resultados do tratamento foram } \\
\text { alcançados. }\end{array}$ \\
\hline $\begin{array}{l}\text { Frez et } \\
\text { al. } \\
(2011)\end{array}$ & $\begin{array}{lr}\text { O objetivo foi } \\
\text { identificar a conduta } \\
\text { em relação à busca do } \\
\text { atendimento } \\
\text { fisioterapêutico, } \\
\text { descrever e quantificar } \\
\text { a satisfação } & \text { dos } \\
\text { usuários após } & \text { o } \\
\text { atendimento } & \text { e } \\
\text { caracterizar } & \text { os } \\
\text { usuários do serviço } \\
\text { ambulatorial rrion } \\
\text { fisioterapia da rede } \\
\text { pública de saúde da } \\
\text { cidade de Foz do } \\
\text { Iguaçu. }\end{array}$ & $\begin{array}{l}\text { A pesquisa } \\
\text { caracterizou-se } \\
\text { como um estudo } \\
\text { transversal, } \\
\text { baseado em um } \\
\text { modelo } \\
\text { epidemiológico } \\
\text { descritivo. } \\
\text { População } \\
\text { avaliada: } \\
\text { Participaram da } \\
\text { amostra } 99 \\
\text { usuários(número } \\
\text { de questionários } \\
\text { respondidos } \\
\text { corretamente), } \\
\text { indivíduos acima } \\
\text { de } 18 \text { anos. }\end{array}$ & $\begin{array}{l}\text { A amostra em sua maioria era } \\
\text { composta por usuários do sexo } \\
\text { feminino, com } 2^{\circ} \text { grau completo e } \\
\text { renda familiar entre quatro e seis } \\
\text { salários mínimos. } \\
\text { predominância dos atendimentos } \\
\text { era da especialidade de ortopedia } \\
\text { e traumatologia. Todos os } \\
\text { pacientes sabiam os seus } \\
\text { diagnósticos. Em relação à } \\
\text { satisfação com os serviços } \\
\text { prestados, os } \\
\text { classificaram usuários } \\
\text { mostrando uma pomo ótima } \\
\text { satisfeita com os serviços que } \\
\text { vem recebendo. }\end{array}$ \\
\hline $\begin{array}{l}\text { Almeida } \\
\text { et al. } \\
(2013)\end{array}$ & $\begin{array}{l}\text { O objetivo foi } \\
\text { identificar o nível de } \\
\text { satisfação do cliente } \\
\text { em um departamento } \\
\text { de fisioterapia na área } \\
\text { pública e analisar as } \\
\text { principais variáveis } \\
\text { que impactam na } \\
\text { qualidade percebida } \\
\text { dos usuários. }\end{array}$ & $\begin{array}{lr}\text { Foi realizado } & \text { um } \\
\text { estudo } & \\
\text { observacional } & \\
\text { transversal. } & \\
\text { População } & \\
\text { avaliada: } & 95 \\
\text { pacientes rom } & \\
\text { idade superior a } 18 \\
\text { anos. }\end{array}$ & $\begin{array}{l}\text { Os pacientes deste serviço de } \\
\text { saúde apresentaram um status } \\
\text { positivo com o tratamento } \\
\text { mostrando que os usuários podem } \\
\text { ser satisfeitos mesmo em um } \\
\text { serviço público de saúde. Houve } \\
\text { influência do nível educacional } \\
\text { no estado de satisfação, pessoas } \\
\text { com mais anos de estudo } \\
\text { apresentaram valores mais baixos } \\
\text { de satisfação. }\end{array}$ \\
\hline
\end{tabular}




\begin{tabular}{|c|c|c|c|}
\hline $\begin{array}{l}\text { Silva et } \\
\text { al. } \\
(2014)\end{array}$ & \begin{tabular}{lr}
\multicolumn{2}{l}{ Avaliar a satisfação de } \\
usuários do serviço de \\
Fisioterapia & (SUS) \\
relatando & os \\
problemas & \\
enfrentados.
\end{tabular} & $\begin{array}{lr}\text { Trata-se de um } \\
\text { estudo qualitativo, } \\
\text { de } \\
\text { descritivo } \\
\text { exploratórior } \\
\text { População } \\
\text { avaliada: } \\
\text { usuários, } \\
\text { indivíduos com a } \\
\text { idade superior a } 18 \\
\text { anos. }\end{array}$ & $\begin{array}{lrr}\text { A razão pela inserção na } \\
\text { fisioterapia foram problemas } \\
\text { osteomioarticulares } \\
\text { neurológicos. Apesar de } \\
\text { divergências, a maioria dos } \\
\text { entrevistados considerou a } \\
\text { fisioterapia do SUS resolutiva. As } \\
\text { questões financeiras } \\
\text { burocráticas foram apontadas } \\
\text { como maiores dificuldades. }\end{array}$ \\
\hline $\begin{array}{l}\text { Cornec et } \\
\text { al. } \\
(2019)\end{array}$ & $\begin{array}{l}\text { O objetivo foi } \\
\text { identificar fatores } \\
\text { associados à satisfação } \\
\text { com a reabilitação } \\
\text { motora (RM) em } \\
\text { crianças e adultos com } \\
\text { paralisia cerebral. }\end{array}$ & $\begin{array}{l}\text { Estudo descritivo } \\
\text { transversal. } \\
\text { População } \\
\text { avaliada: } 1.010 \\
\text { participantes sendo } \\
354 \text { crianças, } 145 \\
\text { adolescentes e } 511 \\
\text { adultos. }\end{array}$ & $\begin{array}{l}\text { Os fatores que diminuíram a } \\
\text { satisfação foram: adolescente, } \\
\text { níveis IV / V do Sistema de } \\
\text { Classificação da Função Motora } \\
\text { Grossa, dor frequente e difícil } \\
\text { acesso a um fisioterapeuta com } \\
\text { treinamento específico em PC. } \\
\text { Fatores que aumentaram a } \\
\text { satisfação foram: presença de um } \\
\text { coordenador de RM, } \\
\text { fornecimento de informações } \\
\text { sobre RM com estabelecimento } \\
\text { de metas e tratamento eficaz da } \\
\text { dor pelo fisioterapeuta. }\end{array}$ \\
\hline
\end{tabular}

Em suma, observa-se que os estudos, destacaram de um modo geral a importância da avaliação da satisfação dos usuários no serviço fisioterapia para melhora na qualidade do serviço.

No trabalho de Machado et al (2008), para verificar a satisfação dos usuários dos serviços de Fisioterapia em Teresina(PI) foi caracterizado o perfil sociodemografico e avaliado por meio de um questionário em relação à agilidade para agendamento da consulta e atendimento, acolhimento, confiança, ambiência, humanização, acessibilidade, eficácia e expectativa sobre os serviços recebidos. Segundo Machado et al (2008) o grau de instrução dos usuários interfere na percepção da satisfação dos usuários, visto que muitos não tem 
conhecimento pleno dos seus direitos e deveres podendo reduzir a capacidade crítica dos pacientes para avaliar com mais objetividade a satisfação quanto ao serviço foi prestado. Silva et al (2014) destaca em seu estudo que nos serviços públicos, os usuários apresentam menor nível de escolaridade e de renda familiar em relação aos serviços privados.

O trabalho de Machado et al (2008) alerta que as pesquisas de satisfação precisam sempre estar em evidencias, pois retratam resultados passageiros e examinam as condições dos serviços que são oferecidos aos usuários naquele período, ou seja, de forma temporária. Essas avaliações das políticas dos sistemas de saúde, precisam de continuo aprimoramento, até mesmo em razão do crescimento e das exigências da população, sob pena de se resultar ineficazes. Estimou-se que fatores socioeconômicos interfiram na escolha do local de atendimento.

No artigo de Frez et al (2011) foi destacado que por ser uma avaliação nunca realizada, o estudo despertou interesse nos administradores responsáveis pelos serviços de fisioterapia. $\mathrm{Na}$ literatura cientifica encontra-se um número pequeno de artigos referentes a percepção dos usuários desses serviços oferecidos pelo SUS e por instituições privadas, por isso esse estudo se mostra importante. Porém foi mencionado um possível viés para estes estudos transversais no quesito da seleção dos sujeitos. No sentido de que aqueles que não responderem o questionário possam ter diferentes percepções e opiniões em relação aos que participaram do estudo. Nesse trabalho a população foi composta por todos os pacientes que receberam alta e o questionário aplicado foi autorrespondido que pode ser considerado um fato positivo, pois dessa forma existe sigilo das informações e diminui o viés de publicação.

Almeida et al (2013) ressaltou que os fatores que realmente predizem a satisfação do paciente no campo da saúde permanece incertos. Essa dificuldade ocorre porque existem muitos perfis diferentes de serviços e usuários de saúde. Apenas a qualidade técnica não é uma ferramenta útil na avaliação dos níveis de satisfação. O paciente está amplamente associado a um aspecto multifatorial, destacando outras variáveis que diz respeito ao terapeuta e os fatores ambientais.

O artigo de Silva et al (2014) menciona que os processos burocráticos de encaminhamento e agendamento são outros fatores importantes que podem interferir na percepção dos usuários e utilização dos serviços de fisioterapia na rede pública. 
No que se refere a particularidade dos indivíduos, pacientes adultos são mais expostos a fatores de riscos como: acidente de transito e de trabalho, doenças crônicas e estresse. Existe uma tendência de consumo maior de álcool e drogas pelos homens do que as mulheres, além de estarem mais susceptíveis a acidentes por causas externas(violência). Aliado a isso, outra característica do sexo masculino é o fato de se considerarem invulneráveis realizando a baixa adesão às práticas de prevenção e não buscarem o cuidado na atenção primaria a saúde, adentrando na rede de saúde pelos serviços de média e alta complexidade na ocorrência de doenças. Isso justifica os achados no estudo de maior incidência masculina e lesões traumato ortopédicas.

Cornec et al (2019) realizaram um estudo para avaliar a satisfação com a reabilitação motora(RM) em pessoas com paralisia cerebral, também identificando as variáveis associadas a essa satisfação. A baixa satisfação com a RM foi associada à idade do adolescente, comprometimento motor grave e alta frequência de dor relatada. $\mathrm{O}$ artigo aponta uma relação para que um paciente avalie sua satisfação como "baixa", e que três condições, que raramente ocorrem juntas em um único serviço, devem acontecer: o paciente precisa ter uma experiência negativa, o problema inicial precisa estar dentro da percepção do paciente como dever de serviço e o paciente deve perceber o prestador de serviço como culpa. O estudo ainda sugeriu que medidas para melhorar a qualidade da assistência à saúde de indivíduos com paralisia cerebral devem se concentrar no aprimoramento do manejo da dor pelo fisioterapeuta, estabelecendo uma aliança terapêutica com o paciente.

Portanto, analisando os estudos dessa revisão narrativa e as abordagens realizadas pelos autores para colher o índice de percepção dos usuários, sugere-se que itens como: gestão do serviço de saúde (acessibilidade, resolutividade), conhecimento dos usuários, contextos biopsicossociais, aliança terapêutica(profissional-paciente) são importantes para a avaliação da percepção de um usuário do serviço de fisioterapia.

\section{CONCLUSÃO}

De modo geral todos os artigos corroboram a ideia de que avaliação da satisfação dos usuários do serviço de fisioterapia é importante para a determinação e qualidade do serviço. 
Essa mensuração aponta as informações do cenário atual, por isso é necessário o aumento periodicamente de pesquisas que visam a avaliação dos serviços prestados. Através desse conhecimento, dos fatores relacionados a satisfação e percepção dos usuários do serviço de fisioterapia, os gestores, administradores e profissionais podem buscar novos modelos de gestão com o foco na melhora da qualidade do serviço.

\section{REFERÊNCIAS}

ALMEIDA, R.S.; NOGUEIRA, L. A.; BOURLLIATAUX-LAIOINE,S.; Análise do nível de satisfação do usuário em um serviço público de fisioterapia. Revista Brasileira de

Fisioterapia, v.17, n.4, p. 328-335, 2013.

ASSUNÇÃO, M. C. F.; SANTOS, I. S.; GIGANTE D. P. Atenção primária em diabetes no sul do Brasil: estrutura, processo e resultados. Revista Saúde Pública, v.35, n. 1, p. 88-95, 2001.

BATESON, J.; HOFFMAN, D. Principios de marketing de serviços: conceitos, estratégias e casos. Pioneira Thompson Learning- São Paulo, ed.2, 2003.

BEATTIE PF, PINTO MB, NELSON MK., NELSON R. Patient satisfaction with outpatient physical therapy: instrument validation. Physical Therapy, v. 32, p. 557-565, 2002.

BRITO, T.A.; JESUS, C.S.; FERNANDES, M.H.; Fatores associados à satisfação dos usuários em serviços de Fisioterapia. Revista Baiana de Saúde Pública, v.36, n.2, p.514526, 2012.

CORNEC, G.; et al. Determinants of motor rehabilitation satisfaction in people with cerebral palsy: a national survey in France (ESPaCe). Annals of Physical and Rehabilitation Medicine - France: Elsevier, 2019. Disponível em:

https://www.sciencedirect.com/science/article/abs/pii/S1877065719301435. Acesso em 10 de abril de 2020.

DONABEDIAN, A. The Quality or Care: how can it be assessed? Jama, v. 260, n.12, p.2330, 1988.

FADEL M. A. V., REGIS FILHO G. I. Percepção da qualidade em serviços públicos de saúde: um estudo de caso. Revista de Administração Pública, v.43, n.1, p. 7-22, 2009.

FERRI, S.M.N.; PEREIRA, M.J.B.; MISHIMA, S.M.; CACCIA-BAVA, M.C.G.;

ALMEIDA, M.C.P.; As tecnologias leves como geradoras de satisfação em usuários de uma 
unidade de saúde da família. Interface - Comunicação, Saúde, Educação, v. 11, n. 23, p. 515-529, 2007.

GURGEL JÚNIOR G. D., VIEIRA M. M. F. Qualidade total e administração hospitalar: explorando disjunções conceituais. Revista Ciência Saúde Coletiva, v. 7, n. 2, p. 325-334, 2002 .

MACHADO, N.P.; NOGUEIRA, L.I.; Avaliação da satisfação dos usuários de serviços de Fisioterapia. Revista Brasileira Fisioterapia, São Carlos, v.12, n.5, p. 401-408, 2008.

MAIA, C.S. et al.; Percepção sobre qualidade de serviços que atendem à saúde da mulher. Revista Ciência Saúde Coletiva, v.16, n.5, p. 2567-2574, 2011.

MENDES A. C. G., ARAÚJO JÚNIOR J. L. C. A., FURTADO B. M. A. S., DUARTE P. O., SANTIAGO R. F., COSTA T. R. Avaliação da satisfação dos usuários com a qualidade do atendimento nas grandes emergências do Recife, Pernambuco, Brasil. Revista Brasileira Saúde Materno Infantil, v. 9, n. 2, p.157- 165, 2009.

MENDONÇA, K.M.P; GUERRA R.O. Desenvolvimento e validação de um instrumento de medida da satisfação do paciente com a fisioterapia. Revista Brasileira Fisioterapia, v.11, n.5, p. 369-376, 2007.

RODRIGUES, R.M.; RAIMUNDO, C.B.; SILVA, K.C; Satisfação dos usuários dos serviços privados de fisioterapia do município de Campos do Goyatacazes/RJ. Revista Perspectivas Online, v.4, n.14, p. 163-180, 2010.

SANTIAGO R. F., MENDES A. C. G., MIRANDA G. M. D., DUARTE P. O., FURTADO B. M. A. S. M., SOUZA W. S. Qualidade do Atendimento nas Unidades de Saúde da Família no município de Recife: a percepção dos usuários. Revista Ciência Saúde Coletiva, v. 18, n. 1, p. 4315-4326, 2013.

SILVA, M. A.; SANTOS, M. L. M.; BONILHA, L. A. S.; Fisioterapia ambulatorial na rede pública de saúde de Campo Grande (MS, Brasil) na percepção dos usuários: resolutividade e barreiras. Revista Comunicação Saúde Educação, v. 18, n. 48, p. 75-86, 2014.

VAITSMAN, J.; ANDRADE, G, R, B.; Satisfação e responsividade: formas de medir a qualidade e a humanização da assistência à saúde. Revista Ciência Saúde coletiva(online), vol.10, n.3, p. 599-613, 2005. 


\section{Declaração de Interesse}

Os autores declaram não haver nenhum conflito de interesse

\section{Financiamento}

Financiamento próprio

\section{Agradecimentos}

O preenchimento desse item é opcional

\section{Colaboração entre autores}

$O$ presente artigo foi escrito pelo $S$. S. $R$. sob orientação do professor J. R. B. G. J., projetado e concluído no Programa Institucional de Bolsas de Iniciação Científica (PIBIC) da Faculdade Dinâmica do Vale do Piranga (FADIP). Ambos os autores cuidaram da parte dissertativa do artigo. 